\title{
Two-Dimensional Ultrasound Receive Array Using an Angle-Tuned Fabry-Perot Polymer Film Sensor for Transducer Field Characterization and Transmission Ultrasound Imaging
}

\author{
Paul Christopher Beard
}

\begin{abstract}
A 2-D optical ultrasound receive array has been investigated. The transduction mechanism is based upon the detection of acoustically induced changes in the optical thickness of a thin polymer film acting as a FabryPerot sensing interferometer (FPI). By illuminating the sensor with a large-area laser beam and mechanically scanning a photodiode across the reflected output beam, while using a novel angle-tuned phase bias control system to optimally set the FPI working point, a notional 2-D ultrasound array was synthesized. To demonstrate the concept, 1-D and 2-D ultrasound field distributions produced by planar 3.5-MHz and focused $5-\mathrm{MHz}$ PZT ultrasound transducers were mapped. The system was also evaluated by performing transmission ultrasound imaging of a spatially calibrated target. The "array" aperture, defined by the dimensions of the incident optical field, was elliptical, of dimensions $16 \times 12 \mathrm{~mm}$ and spatially sampled in steps of $0.1 \mathrm{~mm}$ or $0.2 \mathrm{~mm}$. Element sizes, defined by the photodiode aperture, of $0.8,0.4$, and $0.2 \mathrm{~mm}$ were variously used for these experiments. Two types of sensor were evaluated. One was a discrete $75-\mu \mathrm{m}$-thick polyethylene terephthalate FPI bonded to a polymer backing stub which had a wideband peak noise-equivalent pressure of $6.5 \mathrm{kPa}$ and an acoustic bandwidth $12 \mathrm{MHz}$. The other was a 40- $\mu \mathrm{m}$ Parylene film FPI which was directly vacuum-deposited onto a glass backing stub and had an NEP of $8 \mathrm{kPa}$ and an acoustic bandwidth of $17.5 \mathrm{MHz}$. It is considered that this approach offers an alternative to piezoelectric ultrasound arrays for transducer field characterization, transmission medical and industrial ultrasound imaging, biomedical photoacoustic imaging, and ultrasonic nondestructive testing.
\end{abstract}

\section{INTRODUCTION}

$\mathrm{T}$ HERE are a number of limitations associated with conventional ultrasound receive arrays composed of discrete piezoelectric elements. For applications such as ultrasound field characterization or phased array imaging, the element size should be small compared to the acoustic wavelength in order to provide an omnidirectional response. For example, element sizes of less then 15 microns would be required to provide a near isotropic response at

Manuscript received June 18, 2004; accepted October 28, 2004. The author is supported by an EPSRC Advanced Fellowship.

The author is with the Department of Medical Physics and Bioengineering, University College London, Gower Street, London WC1E 6BT, UK (e-mail: pbeard@medphys.ucl.ac.uk).
$10 \mathrm{MHz}$. Achieving adequate signal-to-noise ratios with piezoelectric elements of this size presents a significant challenge due to the falloff in sensitivity with decreasing element area. A further requirement is that the interelement spacing is less than one half wavelength in order to fulfill the spatial Nyquist criterion. For sampling over even a relatively modest $\left(\sim 1 \mathrm{~cm}^{2}\right)$-sized 2 -D aperture, this necessitates a densely packed array composed of several thousand elements. The need to incorporate a pair of connecting leads and often a preamplifer for each element within the footprint of the array head poses formidable fabrication difficulties and cost implications. Additionally, the close proximity of elements in a dense array can lead to electrical crosstalk due to capacitative coupling between the electrical connection.

A solution may lie in the use of optical techniques whereby the incident acoustic field distribution is mapped, via an appropriate sensor transduction mechanism, onto an optical field. The spatial discretization of the detection process can therefore be removed from the acoustic detection plane to a remotely located high-density array of optical detectors such as a photodiode or charge-coupled device (CCD) array. This offers significant advantages in terms of the spatial sampling of the acoustic aperture. Specifically, substantially smaller (in principle, down to the optical diffraction limit of a few micrometers) element sizes and interelement spacings than can be achieved with piezoelectric arrays are possible. Furthermore, neararbitrary array aperture sizes and geometries can readily be achieved by adjusting the geometry of the optical field that addresses the sensor. The availability of relatively inexpensive CCD and photodiode arrays can also overcome the fabrication difficulties and high cost of piezoelectric arrays. Despite these important advantages, optical methods become truly compelling as an alternative to piezoelectric arrays only if they can provide adequate wideband detection sensitivity. This depends upon the specific optical transduction mechanism employed.

Several approaches have been investigated for mapping ultrasound fields. The detection of acoustically induced changes in optical reflectance at a glass-liquid interface for 2-D photoacoustic imaging [1] has been demonstrated. A system based upon frustrated total internal reflection due 
to the acoustically induced displacement of silicon nitride membranes has been investigated for transmission ultrasound imaging [2]. More sensitive methods tend to employ interferometry. These include the detection of acoustically induced displacements across the surface of a pellicle [3], using a remote confocal Fabry-Perot receiving interferometer and the detection of changes in the optical thickness of multilayer dielectric stack [4], glass [5], and polymer film [6], [7] Fabry-Perot interferometers (FPI). The last of these has been shown unequivocally to provide a wideband high sensitivity that is at least comparable to that of piezoelectric polyvinylidene fluoride (PVDF) transducers [7]-[9]. The versatility and practicality of this approach has been demonstrated by the development of a range of single point fiber-optic probe devices fibre optic sensors for photoacoustic [10] and ultrasound measurement applications [6], [9], and, more recently, a 2-D planar ultrasound array [11] has been developed for biomedical photoacoustic imaging [12], [13]. In this paper we expand upon the use of the Fabry-Perot (FP) polymer film sensor in an array configuration by providing a detailed description of the instrumentation and its operating principles, performance, and specific application to transducer field characterization and transmission ultrasound imaging.

Section II describes the design and fabrication of the FP polymer film sensor head and the optical system used to interrogate and map its output. Section III describes the acoustic performance of the system, and in Section IV its application to mapping the output of planar and focused PZT ultrasound transducers is described. In Section V the use of the system for transmission ultrasound imaging is demonstrated.

\section{FP Ultrasound Array: Design and Operating Principles}

The underlying principle is one in which an FP polymer film sensing interferometer is illuminated by a largediameter continuous-wave $(\mathrm{CW})$ laser beam and the reflected optical beam is directed onto a photodiode mounted on a PC-controlled $x-y$ scanning stage. An incident ultrasound wave modulates the optical thickness of the polymer film FPI, producing a small optical phase shift ( $<150 \mathrm{mrad})$. This is linearly converted, via the FPI transfer function (ITF), the relationship between reflected intensity and optical phase, to a corresponding reflected intensity modulation. By mechanically scanning the photodiode over the reflected output beam of the FPI, the lateral and temporal distribution of the incident acoustic field can therefore be mapped. In this way the system can be regarded as synthesizing an ultrasound array, the aperture of which is defined by the dimensions of the scan region and the element size and interelement spacing by the sensitive area of the photodiode and the scan increment, respectively. The experimental realization of this approach comprises three essential components: (1) the FP sensor head (Section II-A), (2) the optical system used to illu-

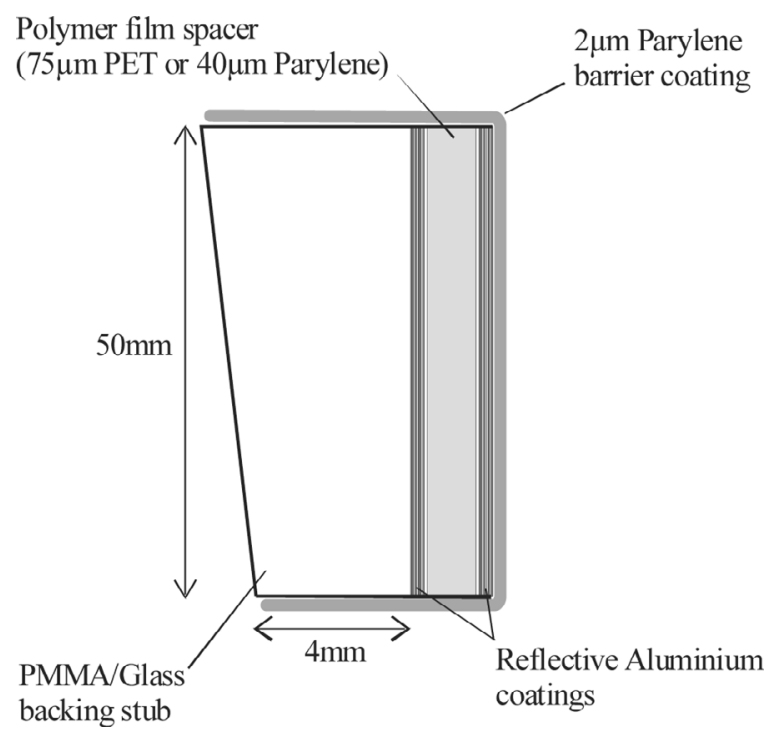

Fig. 1. Fabry-Perot polymer film sensor head.

minate the sensor and map its output (Section II-B), and (3) the angle-tuned phase bias control scheme for setting the working point of the FPI (Section II-C).

\section{A. FP Sensor Head}

A schematic of the sensor head design is shown in Fig. 1. The Fabry-Perot sensing interferometer consists of a polymer film spacer with reflective aluminum coatings on either side. This is backed by a 4 -mm-thick glass or PMMA (polymethyl methacrylate) stub of lateral dimensions $50 \mathrm{~mm} \times 30 \mathrm{~mm}$. A wedge is formed on the optical input side of the backing stub to eliminate parasitic interference between the light reflected from the FPI and that from the front face of the stub. A 2- $\mu$ m-thick protective polymer layer (Parylene C, Specialty Coating Systems, Indianapolis, IN) is deposited over the structure to prevent damage to the external reflective aluminium coating due to water ingress. The following two types of sensor head based on this design were evaluated.

1. 75- $\mu \mathrm{m}$ Polyethylene Terephthalate FPI: The FPI was formed by depositing a partially reflective aluminium coating onto one side of a discrete $75-\mu \mathrm{m}$-thick polyethylene terephthalate (PET) film of refractive index $n=1.64$ and a near-fully reflective aluminium coating on to the other. The film was then bonded, using an optical adhesive (Norland optical adhesives: NOA 68, Norland Products, Inc., Cranbury, NJ), on to a PMMA backing stub. The PET film, the cured adhesive, and the PMMA stub all have similar acoustic impedances [7]. This configuration minimizes the acoustic reflections at the PET film/adhesive/backing stub interfaces, enabling broadband uniform frequency response characteristics to be obtained. This approach provides an inexpensive, flexible, and rapid sensor fabrication method for development and prototyping purposese.g., for evaluating different mirror reflectivities, film thicknesses, and backing materials. However, the process of 
bonding the PET film to the backing stub can lead to variations in the thickness of the cured adhesive layer introducing curvature or distortion in the PET film, particularly for thinner $(<50 \mu \mathrm{m})$ and therefore less rigid films. This distorts the reflected optical beam and therefore the lateral spatial fidelity of the acoustic measurement. The finite thickness of the adhesive layer can also reduce the bandwidth and introduce undesirable resonances in the acoustic frequency response when using a rigid backing material such as glass [7]. For these reasons the following alternative fabrication method in which the polymer film is directly deposited onto the backing stub was also investigated. The free spectral range (FSR) of this sensor is $5.5 \mathrm{~nm}$ at $850 \mathrm{~nm}$.

2. 40- $\mu m$ Parylene FPI: This sensor head was fabricated by depositing a partially reflective aluminium coating directly onto a glass backing stub. This was followed by the deposition of a $40-\mu \mathrm{m}$-thick polymer (Parylene C) layer of refractive index $n=1.64$. This polymer, one of the poly-para-xylylenes, is deposited by forming a monomer gas and introducing it into a vacuum chamber at room temperature. It then condenses and polymerizes on any exposed surface where it forms a highly conformal transparent coating with excellent uniformity of thickness and surface finish [14]. The second, near fully reflective, aluminum coating was deposited on top of the Parylene layer. By directly depositing the polymer film onto the backing stub in this way, the potential problems of distortion in the reflected beam and frequency response limitations (for a rigid backed configuration) due to an adhesive layer can be avoided. The highly uniform optical thickness of the deposited polymer film $(\sim \lambda / 10$ over $1 \mathrm{~cm}$ at $633 \mathrm{~nm}[15])$ also provides for a higher quality FPI with greater fringe visibility and finesse than can be achieved with a PET FPI. Additionally, the use of all vacuum deposition methods enables the sensor head to be inexpensively fabricated in large quantities with high repeatability. The FSR of this sensor is $2.9 \mathrm{~nm}$ at $850 \mathrm{~nm}$.

\section{B. Optical System}

A schematic of the optical system is shown in Fig. 2. The expanded collimated elliptical output beam (of dimensions $16 \times 12 \mathrm{~mm}$ ) of a $70 \mathrm{~mW}, 850 \mathrm{~nm}$, thermally stabilized distributed Bragg reflector laser diode operating in constant current mode is incident, via a PC-controlled precision galvanometer mirror and lenses L1 and L2, on the FP sensor head. The beam reflected from the sensor is then directed, via a beamsplitter and L3, onto a 25$\mathrm{MHz}$ dc-coupled silicon photodiode/transimpedance amplifier configuration mounted on a PC-controlled $x-y$ scanning stage. The diameter of the sensitive region of the photodiode was $0.8 \mathrm{~mm}$, reduced to $0.4 \mathrm{~mm}$ and $0.2 \mathrm{~mm}$ for the experiments described in Section IV by placing circular apertures in front of it. The high-pass $(>300 \mathrm{kHz})$ filtered voltage output of the photodiode $\mathrm{d} v_{\mathrm{ac}}$ is recorded using a 500-MHz digitizing oscilloscope (DSO) and used to measure the small time-varying reflected intensity modulation due to an incident acoustic wave. The photodiode output is also connected to a low-speed $(20 \mathrm{kHz})$ A-D card within the PC. The resulting digitized signal is referred to as the low-pass filtered photodiode output $\mathrm{V}_{\mathrm{dc}}$ and is used to record the reflected intensity variations produced by the rotation of the galvanometer mirror as part of the procedure to set the optimum operating point of the FPI as described in Section II-C. Lenses L1, L2, and L3 are each of focal length $59 \mathrm{~mm}$ and aperture $25 \mathrm{~mm}$.

In principle, to map an acoustic field, the photodiode can be mechanically scanned over the reflected output beam of the FPI and the acoustic signal captured by the DSO at each point of the scan. However, a difficulty arises in that the sensitivity of the FPI varies from point to point due to changes in the optical thickness of the polymer film. That is to say, the phase bias or working point of the FPI, and therefore the slope of the ITF which provides a measure of sensitivity, is position dependent. This can be seen in Fig. 2 of [15] which shows a map of the acoustic sensitivity of the $40-\mu \mathrm{m}$-thick Parylene film sensor. Over a $39 \times 25 \mathrm{~mm}$ area there are just two narrow contours of a few millimeters' width of high sensitivity - elsewhere the sensor is largely insensitive.

To overcome this problem and interrogate any point on the FPI with optimum sensitivity, the optical pathlength of the FPI, and hence its phase bias, is controlled by varying the angle of incidence of the illuminating beam. The theory and practical implementation of this approach are described in detail in [15]. Briefly, however, it is achieved using the optical subsystem shown in Fig. 2 comprising lenses L1 and L2 (separated by a distance equal to twice their focal lengths) and the galvanometer mirror which is situated in the back focal plane of L1. The FP sensor head is positioned in the front focal plane $\mathrm{P} 1$ of $\mathrm{L} 2$ and its reflected output beam equivalently reversed through the system by directing it through L3 and imaging it onto the photodiode situated in the focal plane (P2) of L3. Changing the angle $\theta_{\text {in }}$ of the input beam using the galvanometer mirror produces an identical change in the angle $\theta$ of the light incident on the sensor without translating it across the surface. Similarly, the reflected FPI output beam that is incident on the photodiode is pivoted about $\mathrm{P} 2$ without translation as $\theta_{\text {in }}$ varied. By ensuring there is no lateral translation in this way, the spatial correspondence between the acoustic detection point at P1 and its corresponding optical detection point at P2 is preserved. Providing $\theta$ can be varied over a sufficiently large range to produce a change in the phase bias of at least $2 \pi$ radians, any point on the illuminated region of the sensor can be interrogated with optimum sensitivity. Two methods of interrogating the sensor using this system are described in the following section.

\section{Phase Bias Control Schemes}

1. Active Phase Bias Control: This approach was used for the transducer field mapping experiments described in 


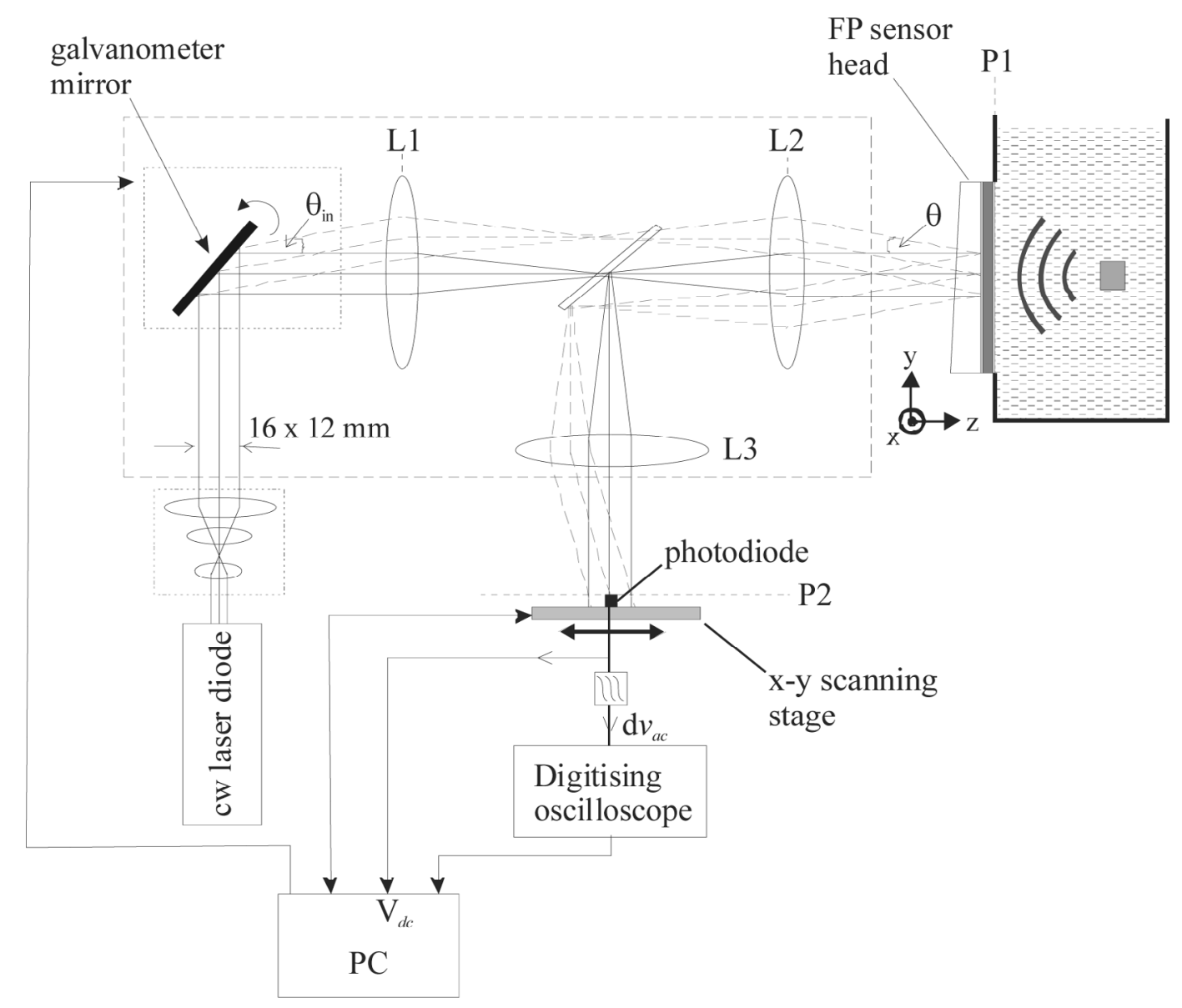

Fig. 2. Experimental optical system setup.

Section IV and involves actively locating the optimum operating point of the FP sensor prior to capturing an acoustic waveform. To implement this, an increasing arccosine voltage function (generated by the PC) is applied to the galvanometer mirror such that the beam incident on the sensor is rotated from normal incidence through an external angle $\theta$ of approximately $11^{\circ}$. The FPI reflectedintensity output is simultaneously monitored by recording the low-pass filtered output of the photodiode, $\mathrm{V}_{\mathrm{dc}}$. Since the angle-induced phase shift is proportional to the cosine of the angle [15], the use of an arccosine angular function means that $V_{\mathrm{dc}}$ is obtained in steps of constant phase and therefore represents the ITF. The derivative of the ITF, which represents the sensitivity of the FPI to an acoustically induced phase shift (the phase sensitivity), is then calculated and the mirror returned to the angle corresponding to the peak value of the ITF derivative. The sensor is now optimally biased for maximum sensitivity and ready to make a measurement.

An automated PC-controlled system based on this approach was implemented running under using Labview (National Instruments Corp., Austin, TX). Fig. 3 shows an example of the system output obtained using the $40-\mu \mathrm{m}$ Parylene film sensor. This shows $\mathrm{V}_{\mathrm{dc}}$, its phase derivative $\mathrm{dV}_{\mathrm{dc}} / \mathrm{d} i$, and the optimum phase bias point $\phi_{\mathrm{o}}$ located by the system. The term $i$ is the index of the PC-generated array containing the arccosinusoidally increasing voltage values that are sequentially applied to the mirror; the horizontal axis in Fig. 3 is therefore scaled in steps of constant phase. For illustrative purpose, a He-Ne laser source operating at $633 \mathrm{~nm}$ was used rather than the $850-\mathrm{nm}$ laser diode in order to show the periodic nature of the ITF in Fig. 3. This is because the longer wavelength of the laser diode did not quite enable a full $2 \pi$ phase shift to be obtained with the maximum $11^{\circ}$ angular range (limited by the focal length and aperture of L1-3) of the system. This was not a significant limitation for the experiments described in Sections IV and V which used the 850-nm laser diode. Within the limited dimensions of the illuminated region of the sensor, the phase bias variations due to changes in optical thickness were generally less than $2 \pi$, enabling the maximum slope of the ITF to still be located. Note that the ITF shown in Fig. 3 is not the usual symmetrical Airy function characteristic of an FPI. Its asymmetric sawtooth shape is due to the fact that the reflection coefficients of the aluminium coatings used to form the FPI mirrors are complex due to absorption [16], [17].

To map the output of the sensor, the photodiode is scanned over the reflected output beam at P2. At each point of the scan, the optimum phase bias $\phi_{\mathrm{o}}$ is located as described above and the time record of the acoustically induced intensity modulation detected by the photodiode (the high pass filtered output $\mathrm{d} v_{\mathrm{ac}}$ ) captured by the DSO and downloaded to the PC. Since the value of the ITF 


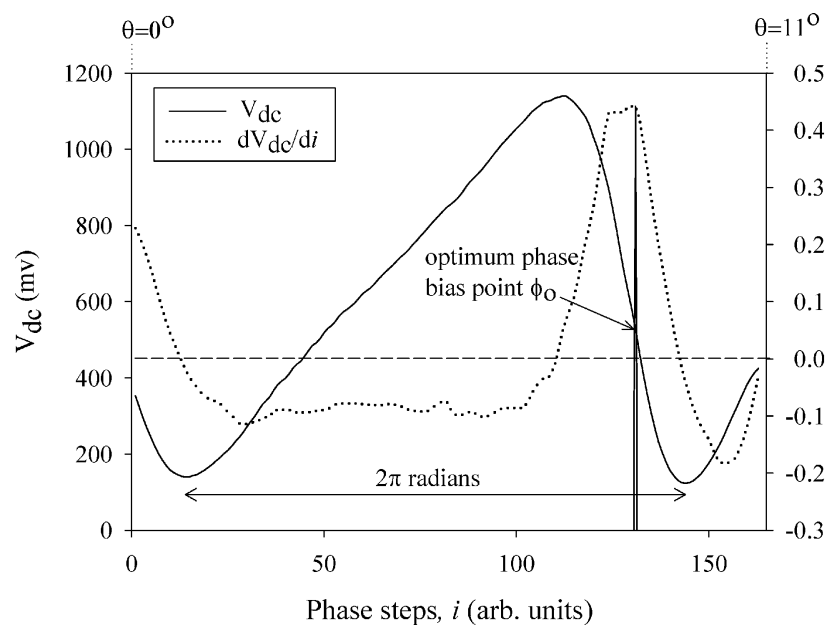

Fig. 3. Output of active phase bias control system obtained by rotating the FPI illuminating beam angle of incidence $\theta$ through $11^{\circ}$ using an arccosine angular function to obtain constant phase steps $i$. Graph shows the FPI transfer function (represented by $\mathrm{V}_{\mathrm{dc}}$ ), its phase derivative $\left(\mathrm{dV}_{\mathrm{dc}} / \mathrm{d} i\right)$, and the optimum operating point $\phi_{\mathrm{o}}$ located by the system. The latter is indicated by the vertical line and corresponds to the peak value of $\mathrm{dV}_{\mathrm{dc}} / \mathrm{d} i$, the point of maximum sensitivity. The FPI was the $40-\mu \mathrm{m}$ Parylene FP sensor, $\lambda=633 \mathrm{~nm}$.

derivative is a measure of the sensitivity, it can be used to correct for variations in the FPI phase sensitivity due, for example, to variations in intensity across the incident illuminating beam or in the reflectivity of the aluminium coatings. To implement this, the acoustic waveform captured at each point of the scan is divided by the peak value of the ITF derivative obtained at that point.

The acquisition time using this system is approximately 3 seconds per scan step. This is made up of three components: (1) the time taken to rotate the galvanometer and locate the optimum operating point: 0.5 seconds [In principle, the inherent speed of the galvanometer would allow this to be reduced to a few tens of milliseconds but was limited in practice by the specific D-A card used. This did not have an on-board buffer and therefore required each of the 160 values in the array containing the arccosinusoidally increasing voltage function to be output sequentially from the Labview control software.], (2) the time for the stepper motor to move an increment: approximately 1 second depending on step size, and (3) the time to acquire and download the acoustic waveform to the DSO: 1.5 seconds.

2. Continuous Phase Bias Scanning: In contrast to the previous method of actively setting the optimum phase bias of the system prior to capture the acoustic signal, this approach involves continuously scanning the phase bias over $2 \pi$ radians and recording the amplitude of a repetitive pulsed acoustic signal at the instant in time it happens to coincides with the optimum phase bias point. To implement this approach, the galvanometer was replaced with a mirror mounted on a dc motor continuously rotating through $360^{\circ}$. As the input beam is swept over the lens L1, the time records of the low- and high-pass filtered photodiode outputs, $\mathrm{V}_{\mathrm{dc}}$ and $\mathrm{d} v_{\mathrm{ac}}$, respectively, were cap-

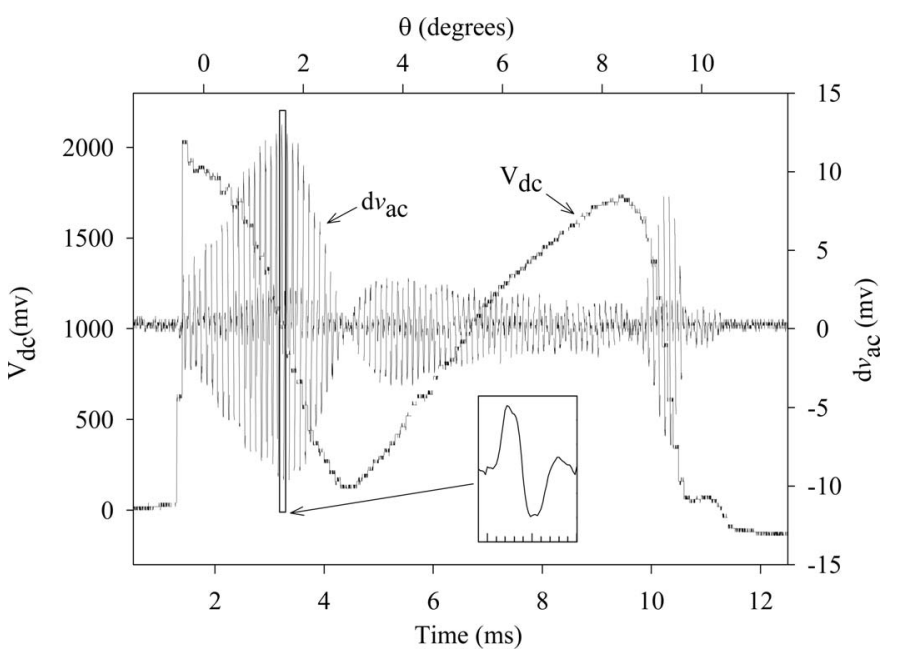

Fig. 4. FPI output obtained using a mirror continuously rotating at $1.43 \mathrm{~Hz}$ and incident acoustic pulses at a repetition frequency of $10 \mathrm{kHz} . \mathrm{V}_{\mathrm{dc}}$ represents the reflected intensity due to the slowly varying change in phase bias due to the rotation of the mirror. The term $\mathrm{d} v_{\text {ac }}$ represents the reflected intensity modulation due to the incident acoustic pulses. Inset shows expanded view over $0.5 \mu \mathrm{s}$ of maximum acoustic signal which occurs at the peak of the ITF phase derivative. The FPI was the $40-\mu \mathrm{m}$ Parylene FP sensor, $\lambda=633 \mathrm{~nm}$.

tured simultaneously on separate channels of the DSO. An example of this is shown in Fig. 4 for a mirror-rotation angular frequency of $9 \mathrm{rad} / \mathrm{sec}(1.43 \mathrm{~Hz})$ and ultrasound pulses emitted by a $3.5-\mathrm{MHz}$ PZT ultrasound transducer operating at a repetition frequency of $10 \mathrm{KHz}$; the FPI was the $40-\mu \mathrm{m}$ Parylene film sensor. The term $\mathrm{V}_{\mathrm{dc}}$ represents the slowly varying reflected intensity due to the angle-induced phase bias variation. Note, however, that due to the constant angular velocity of the mirror, $\mathrm{V}_{\mathrm{dc}}$ is obtained in steps of constant angle, not constant phase as in Fig. 3. Thus it does not represent the "true" ITF as does Fig. 3 although the underlying features of the ITF, such as its periodic nature and asymmetry, are still apparent. The time record of $\mathrm{d} v_{\mathrm{ac}}$ shows the individual acoustic pulses detected over the period of the angle-induced phase bias variation, the inset in Fig. 4 shows one of these acoustic pulses. The acoustic signals are amplitude modulated by the ITF phase derivative thus falling to zero at the turning points of $\mathrm{V}_{\mathrm{dc}}$ and rising to a maximum at the point of maximum slope on the ITF.

To make practical use of this method, the horizontal axis of Fig. 4 could be rescaled using the cosine relationship between angle and phase [15] to obtain a linear phase scale. The true ITF and its phase derivative could then be obtained and the acoustic signal that coincided with the maximum value of the derivative extracted. However, if only the signal amplitude rather than a time resolved measurement is required, a peak detect algorithm can simply be applied to the time record of $\mathrm{d} v_{\mathrm{ac}}$. This will automatically recover the amplitude of the signal at the instant in time the sensor is at its optimum phase bias. The latter approach was used to obtain the transmission ultrasound image shown in Section V. 
In summary then, to map the acoustic signal amplitude using this method, the photodiode is scanned across the reflected output beam of the sensor. At each point of the scan, the time records of $\mathrm{V}_{\mathrm{dc}}$ and $\mathrm{d} v_{\mathrm{ac}}$ over the period the beam is sweeping across the input lens L1 are captured by the DSO. Peak detect measurements on both time records are then made by the DSO and the two values downloaded to the PC. The peak detect measurement of $\mathrm{d} v_{\text {ac }}$ provides the desired acoustic signal amplitude. The peak-peak value of $\mathrm{V}_{\mathrm{dc}}$ is approximately proportional to the peak value of the ITF slope and can therefore be used to correct for variations in sensitivity by dividing into the acoustic signal amplitude as in the previous section. The acquisition time per scan step is similar to that of the previous section, being dominated by the stepper motor speed and the download time to the PC. The advantage of the phase bias scanning approach, compared to the active phase bias control method of Section II-C,1, is that it avoids the expense of a precision galvanometer and the need for a $\mathrm{PC}$ control system.

\section{Acoustic Performance}

The following subsections describe the acoustic performance of the system in terms of the essential parameters required to specify an ultrasound receive array, namely, sensitivity, linearity, frequency response, element size, array aperture, and interelement sensitivity variations.

\section{A. Detection Sensitivity}

The detection sensitivity or noise-equivalent pressure (NEP) is defined as the acoustic pressure that provides a system signal-to-noise ratio of unity in the low-frequency limit [7] $\lambda_{\mathrm{a}} \gg l$ where $\lambda_{\mathrm{a}}$ is the acoustic wavelength and $l$ the FPI thickness. The NEP therefore represents the minimum detectable acoustic pressure and is given by

$$
N E P=\frac{N}{S}
$$

where $\mathrm{S}$ is the sensor sensitivity and is defined as the reflected optical power modulation per unit acoustic pressure $(\mu \mathrm{W} / \mathrm{MPa})$ at the FPI optimum phase bias $\phi_{\mathrm{o}}$, and $\mathrm{N}$ is the minimum detectable optical power modulation reflected from the sensor over a specified measurement bandwidth and is a function of the noise characteristics of the laser source and the photodiode/transimpedance amplifier configuration; it is the noise characteristics of the latter that dominates in this system. The $\mathrm{S}$ depends upon a range of fixed parameters such as the FPI mirror reflectivities (which define the shape of the ITF), the thickness and elastic and photoelastic properties of the polymer film, and the acoustic impedance of the backing stub. Also, S, and to a lesser extent $\mathrm{N}$, depend upon the incident optical intensity and this varies from point to point across the illuminating beam which has a nominally Gaussian spatial distribution. For the NEP values given below for the two types of sensor used, the photodiode (with a 0.4-mm circular aperture in front of it) was positioned at the center of the reflected output beam where the intensity is at a maximum. Under these conditions, the power falling on the photodiode was approximately $50 \mu \mathrm{W}$.

To obtain S, the reflected intensity modulation produced by the output of a calibrated PZT ultrasound source operating at a nominal center frequency of $3.5 \mathrm{MHz}$ was measured. To obtain N, the peak-to-peak output noise voltage of the photodiode was measured over a $25-\mathrm{MHz}$ bandwidth. No signal averaging was used.

For the $75-\mu \mathrm{m}$ PET sensor, the peak NEP was measured to be $6.5 \mathrm{kPa}$ over a $25-\mathrm{MHz}$ measurement bandwidth. Under the same measurement conditions the corresponding value for the $40-\mu \mathrm{m}$ Parylene sensor was $8 \mathrm{kPa}$. These NEP values are comparable to those obtainable with a PVDF detector of diameter $200 \mu \mathrm{m}$ [9], and recent work has shown that there is the potential to improve upon them by at least an order of magnitude [8].

\section{B. Linearity}

Assuming the elastic limits of the polymer film and the linear operating range of the photodiode are not exceeded, the upper limit of linear acoustic detection is determined by the phase range around $\phi_{\mathrm{o}}$ over which the ITF is linear. For the ITF shown in Fig. 3, the linear phase range is $0.26 \mathrm{rad}$, obtained by estimating the phase excursion over which the ITF departs from a linear relationship by $10 \%$. Using a value for the acoustic phase sensitivity of $0.12 \mathrm{rad} / \mathrm{MPa}$ (based on a previously reported value of $0.15 \mathrm{rad} / \mathrm{MPa}$ for $50-\mu \mathrm{m}$-thick rigid-backed Parylene film [9]), this corresponds to a linear (to within 10\%) pressure range of 2.2 MPa for the $40-\mu \mathrm{m}$ Parylene sensor at $850 \mathrm{~nm}$. Although having nominally similar mirror reflectivities as the 40- $\mu \mathrm{m}$ Parylene sensor, the phase derivative of the ITF at $\phi_{\mathrm{o}}$ of the $75-\mu \mathrm{m}$ PET sensor was somewhat lower, resulting in a larger linear phase range of $0.48 \mathrm{rad}$. Using a value for the acoustic phase sensitivity of $0.15 \mathrm{rad} / \mathrm{MPa}$ (based on a previously reported value of $0.1 \mathrm{rad} / \mathrm{MPa}$ for a 50- $\mu \mathrm{m}$ water-backed PET film [7]), this corresponds to a linear (to within 10\%) pressure range of 3.2 MPa. Since the upper limit of linear detection scales with the thickness of the polymer sensing film and the reflectivity finesse, there is scope to increase it. For example, as in the optical fiber hydrophone configuration described in [10], a $25-\mu \mathrm{m}$ rigidbacked FPI sensor with a low-finesse ITF would provide a linear operating range to $11 \mathrm{MPa}$.

\section{Frequency Response}

Assuming the sensor operates predominantly in thickness mode (i.e., no radial resonances or longitudinaltransverse mode coupling), the bandwidth for a normally incident plane wave is determined by the thickness and the speed of sound of the polymer film and the acoustic impedance of the backing material. The uniformity of response is determined by the acoustic impedance mis- 


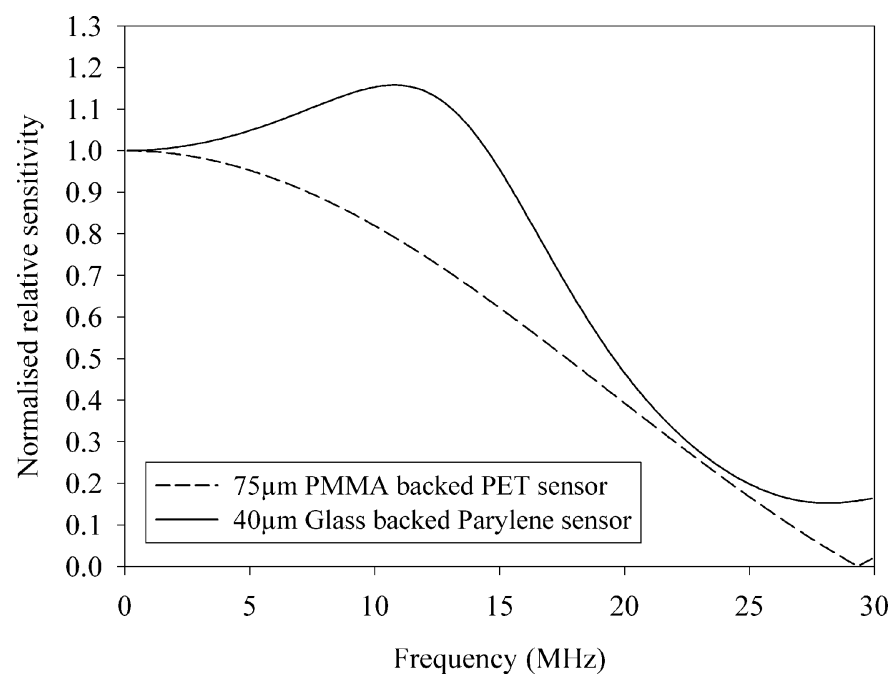

Fig. 5. Predicted normalized frequency response characteristics of 40- $\mu \mathrm{m}$ Parylene and $75-\mu \mathrm{m}$ PET sensors.

matches at the boundaries of the film, on one side due to the backing, on the other due to the surrounding water. In general, polymer films have an acoustic impedance close to water and uniform broadband response characteristics can therefore be expected. This can be seen in Fig. 5 which shows the predicted frequency response characteristics of the two sensors for a normally incident plane wave. These were obtained using an experimentally validated analytic model [7] that calculated the frequency-dependent mean distribution of stress across the polymer film thickness by the summation of acoustic reflections within the film. The glass-backed $40-\mu \mathrm{m}$ Parylene sensor, shows a small $\lambda / 4$ resonance at $11.5 \mathrm{MHz}$ characteristic of a rigid-backed configuration. Thereafter the response falls off to a minimum at $27.5 \mathrm{MHz}$ when the acoustic wavelength $\lambda_{\mathrm{a}}$ is equal to twice the thickness $l$ of the polymer film. The $3-\mathrm{dB}$ bandwidth is $17.5 \mathrm{MHz}$. The impedance-matched nature of the PMMA-backed 75- $\mu \mathrm{m}$ PET sensor means that there is no resonance and the response rolls off smoothly to a minimum at $29.3 \mathrm{MHz}$ when $\lambda_{\mathrm{a}}=l$. The $3-\mathrm{dB}$ bandwidth of this sensor is $12.5 \mathrm{MHz}$.

\section{Element Size}

To a first approximation, the element size is defined by the dimensions of the optical field addressing the sensorin this case the area of aperture in front of the photodiode. Clearly there will be some limit to this assumption. Acoustically induced thickness changes beyond the optically defined area of an "element" will to some extent contribute to its output, resulting in an apparent increase in the element size. The lower limit has yet to be determined categorically although previous directivity measurements [9] of a sensor illuminated by a 6 - $\mu$ m-diameter beam indicate that effective radii down to at least $50 \mu \mathrm{m}$ (and possibly significantly lower) can be achieved. In the experiments reported in this paper the photodiode aperture was always greater than $200 \mu \mathrm{m}$ (to ensure that sufficient laser power was incident on the photodiode to maintain adequate detection sensitivity), so it is considered that the lower limit of the effective element size was not approached.

\section{E. Array Aperture and Interelement Sensitivity Variations}

The notional array aperture is the acoustically sensitive region of the sensor which is defined by the dimensions of the optical beam incident on the FPI. To measure the extent and distribution of sensitivity over this region, plane waves emitted by a $2.5-\mathrm{cm}$ diameter $3.5-\mathrm{MHz} \mathrm{PZT}$ planar transducer were directed at the sensor; the sensortransducer separation was $5.6 \mathrm{~cm}$. Given the large diameter of the transducer, it is assumed that the amplitude across the emitted plane wavefront over the illuminated region of sensor was constant. The photodiode was line scanned vertically $(y)$ and horizontally $(x)$ through the center of the FPI output beam and the acoustic signal amplitude recorded at each point. At each point of the scan, the active phase bias control method described in Section II-C,1 was used to optimally bias the sensor and obtain a value of the ITF derivative.

In Fig. 6, the dashed lines represent the raw detected signal amplitude (i.e., without dividing by the ITF derivative to correct for sensitivity variations) as a function of position for the vertical and horizontal scans. These follow the profile of the illuminating beam. The solid lines in Fig. 6 represent the corrected sensitivity profiles, obtained by dividing the signal amplitude obtained at each point by the corresponding value of the ITF peak derivative as described in Section II-C,1. This procedure compensates for sensitivity variations due to the intensity profile of the illuminating beam, resulting in a relatively flat sensitivity distribution that falls off only at the extremities of the beam where the reflected intensity has fallen below the photodiode detection limit. From these "corrected" profiles, it can be seen that the array aperture is a $15 \times 12 \mathrm{~mm}$ ellipse. The variations over the corrected profiles provide a measure of the notional interelement sensitivity variations, approximately $\pm 15 \%$. This is a relatively large figure and is thought to be due to position-dependent distortions in the ITF, due to lens and other aberrations in the optical system [15]. This leads to the optimum phase bias being incorrectly set with a consequent error in the value of the recovered peak ITF derivative, the latter exacerbating the problem given its use as a correction factor.

\section{Transducer Field Mapping}

\section{A. One-Dimensional Scans of Planar 3.5-MHz Transducer}

The system was used to map the output of a pulsed 3.5-MHz PZT planar transducer of diameter $25 \mathrm{~mm}$. The peak positive acoustic pressure output of the transducer was approximately $0.05 \mathrm{MPa}$. The sensor head was the $40-\mu \mathrm{m}$ Parylene sensor. An aperture of $0.4 \mathrm{~mm}$ was placed in front of the photodiode. The transducer was aligned 

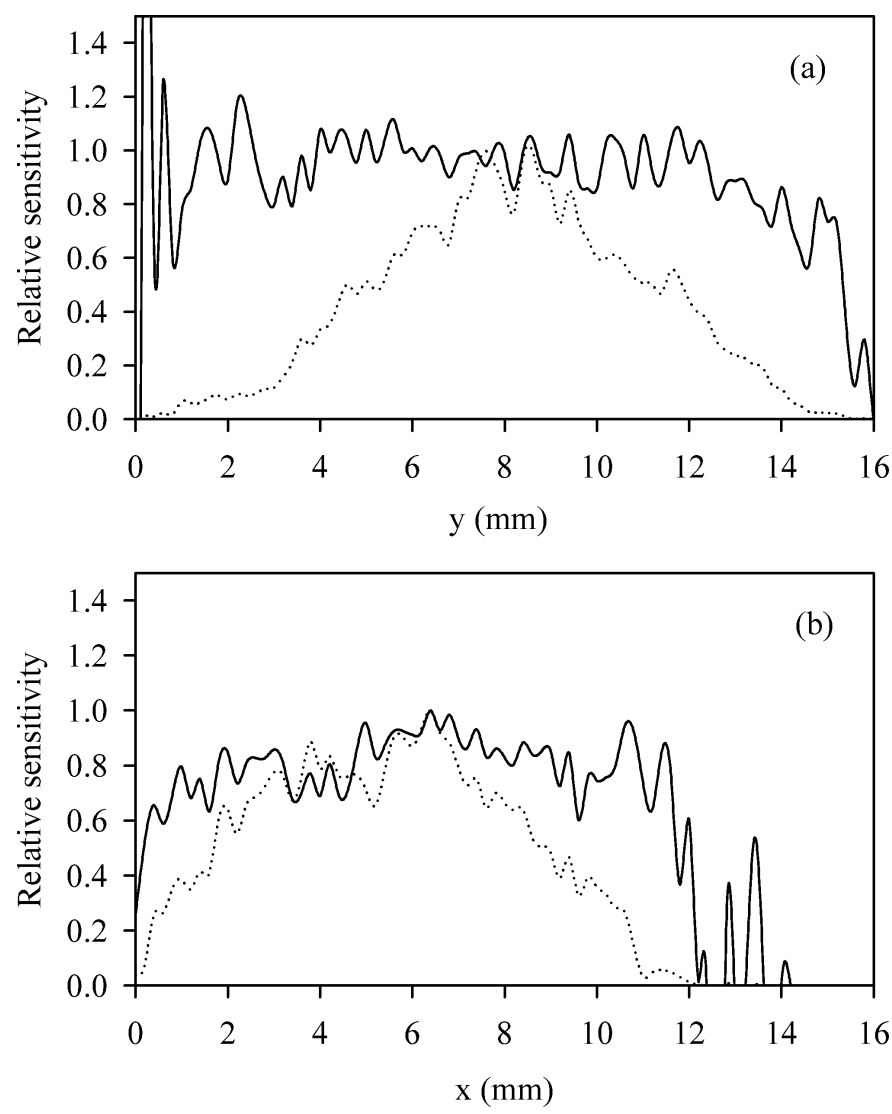

Fig. 6. (a) Vertical and (b) horizontal sensitivity distributions. The dashed lines represent the "raw" signal amplitude as a function of position. The solid lines represent the "corrected" sensitivity profiles obtained by dividing the signal amplitude by the peak ITF derivative. Photodiode aperture $=0.4 \mathrm{~mm}$. The FPI was the $75-\mu \mathrm{m}$ PET sensor.

parallel to the sensor $x-y$ detection plane in a water bath and the photodiode scanned in steps of $0.1 \mathrm{~mm}$ along a line of length $16 \mathrm{~mm}$ in the $y$ direction that passed through the transducer axis. At each point of the scan, the active phase bias control method described in Section II-C,1 was used to set the optimum working point of the FP sensor. A portion of the time record of the high-pass filtered photodiode output $\mathrm{d} v_{\mathrm{ac}}$, which represents the acoustic waveform, was then captured by the DSO, using a delayed trigger, and downloaded to and stored by the PC. The resulting set of detected acoustic signals $p(y, t)$ were then mapped to a linear grayscale and displayed as a 2-D image as shown in Fig. 7. This shows linescans obtained for three different transducer-sensor separations: $z=5.8 \mathrm{~cm}, z=3.46 \mathrm{~cm}$, and $z=1.5 \mathrm{~cm}$.

Each of the images in Fig. 7 is characterized by an initial tripolar planar wavefront $\mathrm{P}$ (seen in the images as three horizontal bands of alternating polarity) that arrives at a time corresponding to the perpendicular distance between the sensor detection plane and the transducer. This planar wavefront is then followed by inverted edge waves $\mathrm{E}$ originating from the circumference of the transducer which appear as a characteristic X-shaped feature in each of the images in Fig. 7. At the center of each scan $(\sim y=8 \mathrm{~mm})$ the photodiode lies on the axis of the transducer. The edge wave contributions from each point around the transducer circumference therefore arrive at the same time producing a signal maximum at the center of the $\mathrm{X}$ feature. As the detector moves off axis, the symmetry is broken and the contributions from different points around the transducer circumference arrive at different times, the temporal extremes of which are indicated by the diagonal lines of the X-shaped feature. The time-of-arrival of the edge wave components relative to that of the initial plane wavefront increases with decreasing transducer-detector distance. This is clearly in evidence in Fig. 7 which shows the center of the X-shaped feature arriving progressively later in time with increasing $z$. In each of the three images a time delayed plane wavefront $\mathrm{R}$ is also in evidence. This is the reflection of $\mathrm{P}$ from the back of the 4-mm-thick glass backing stub of the sensor.

\section{B. Two-Dimensional Scan of a Focused Transducer}

The spatial and temporal output of a pulsed $5-\mathrm{MHz}$ focused PZT transducer of diameter $29 \mathrm{~mm}$ and focal length $63.5 \mathrm{~mm}$ was mapped using a similar approach to that described in the previous section. This time, however, the photodiode was scanned over a $6 \times 6$ - $\mathrm{mm}$ area in the $x-y$ plane in steps of $0.2 \mathrm{~mm}$ to obtain the 2-D distribution of the incident acoustic field. Thus the recorded data set was a $3 \mathrm{D}$ array $p(x, y, t)$, representing the distribution of the incident acoustic pressure in the $x$ - $y$ detection plane as a function of time. The 2-D scans were performed with the transducer at three different distances from the sensor: $z=8.2 \mathrm{~cm}, z=7.7 \mathrm{~cm}, z=6.6 \mathrm{~cm}$. The photodiode aperture was $0.2 \mathrm{~mm}$ and the $75-\mu \mathrm{m}$ PET sensor was used for these experiments.

The results are shown in Fig. 8. The upper three images show the $x-y$ pressure distributions for the three transducer-sensor separations. The diameter of the circular pressure distributions represent the full-width at halfmaximum values of the acoustic beamwidth $\mathrm{W}$ for each value of $z$. These are, for decreasing $z, W=3.9 \mathrm{~mm}$, $W=2.7 \mathrm{~mm}$, and $W=1.2 \mathrm{~mm}$, and are in approximate agreement with calculated beam profiles based upon the geometry and frequency output of the transducer. The lower three images show the corresponding pressure time records along a line in the $x$-direction through the center of each of the $x-y$ pressure distributions. These show the decreasing curvature of the wavefronts and beamwidth as the source-sensor separation approaches the transducer focus culminating in a minimum beamwidth and near planar wavefront at the focus.

\section{Transmission Ultrasound Imaging}

To demonstrate the principle of using the system for transmission ultrasound imaging, a plastic mesh target of the dimensions shown in Fig. 9 was placed between the sensor head and the planar $3.5-\mathrm{MHz}$ transducer. The photodiode (no aperture was used) was scanned over a $10.5 \times 10.5$ $\mathrm{mm}$ area of the sensor output beam in steps of $0.1 \mathrm{~mm}$. 

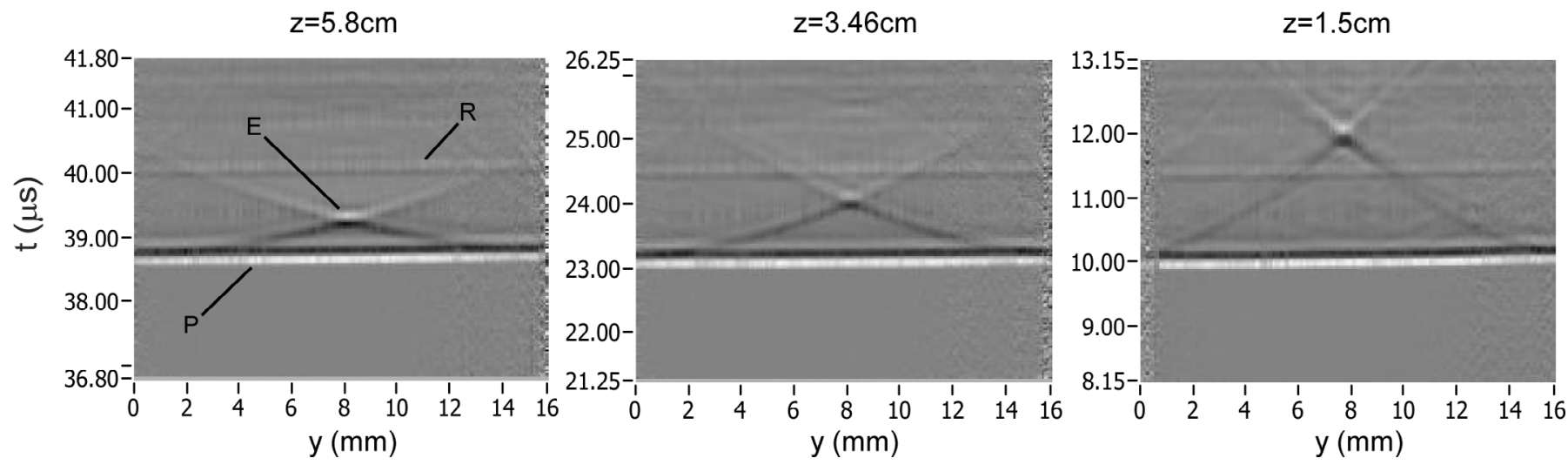

Fig. 7. Vertical linescans of the output of a pulsed 3.5-MHz planar PZT transducer for three transducer-sensor separations $z(z=5.8 \mathrm{~cm}$, $z=3.46 \mathrm{~cm}, z=1.5 \mathrm{~cm}$ ) obtained using the $40-\mu \mathrm{m}$ Parylene sensor. $\mathrm{P}=$ initial plane wave, $\mathrm{E}=$ edge wave component, $\mathrm{R}=$ reflection from back surface of $4-\mathrm{mm}$ glass backing stub; linescan length $=16 \mathrm{~mm}$, scan increment dy $=0.1 \mathrm{~mm}$, photodiode aperture $=0.4 \mathrm{~mm}$.
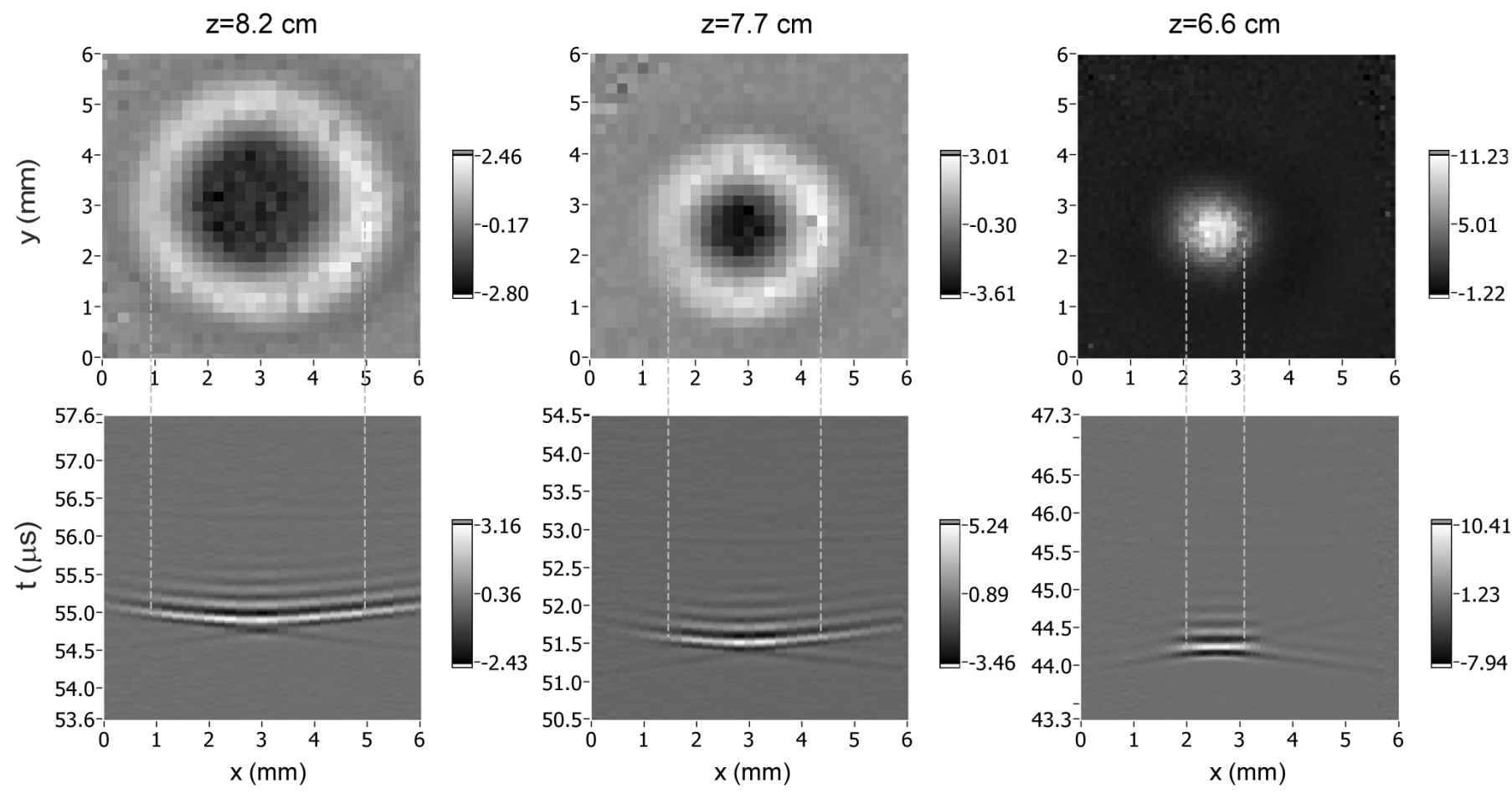

Fig. 8. Two-dimensional scans of the output of a pulsed 5-MHz focused PZT transducer for three transducer-sensor separations $z(z=8.2 \mathrm{~cm}$, $z=7.7 \mathrm{~cm}, z=6.6 \mathrm{~cm})$ obtained using the $75-\mu \mathrm{m}$ PET sensor. The upper row of images shows the lateral pressure distributions $p(x, y)$ for each $z$. The lower row of images shows the corresponding pressure time records $p(x, t)$ along a line in the $x$-direction through the center of each of the $x-y$ pressure distributions. Scan area $=6 \mathrm{~mm} \times 6 \mathrm{~mm}$, scan increments $d x=d y=0.2$ mm, photodiode aperture $=0.2 \mathrm{~mm}$.

The continuous phase bias scanning method described in Section II-C,2 in which a continuously rotating mirror was used in placed of the galvanometer mirror was employed to interrogate the sensor. Unlike the transducer mapping experiments described in the previous section, only the amplitude of the detected signal was required. Therefore the method of applying a peak detect algorithm to the time record of the high-pass filtered photodiode output $\mathrm{d} v_{\mathrm{ac}}$ during the period the input beam is swept across L1 to extract the signal amplitude as outlined in Section II-C,2 was employed. Fig. 9 shows a map of the detected signal amplitude, showing clearly the spatial characteristics of the target. The lateral resolution, limited by the photodiode dimensions is $0.65 \mathrm{~mm}$. Using a detector array, this simple method of sensor interrogation could be employed to realize an acoustic camera for applications such as transmission ultrasound imaging or spatial field mapping where a time-resolved measurement is not required.

\section{Conclusions}

An optical method for mapping the spatial and temporal distribution of ultrasound fields has been demonstrated. This has shown that it is possible to synthesize 

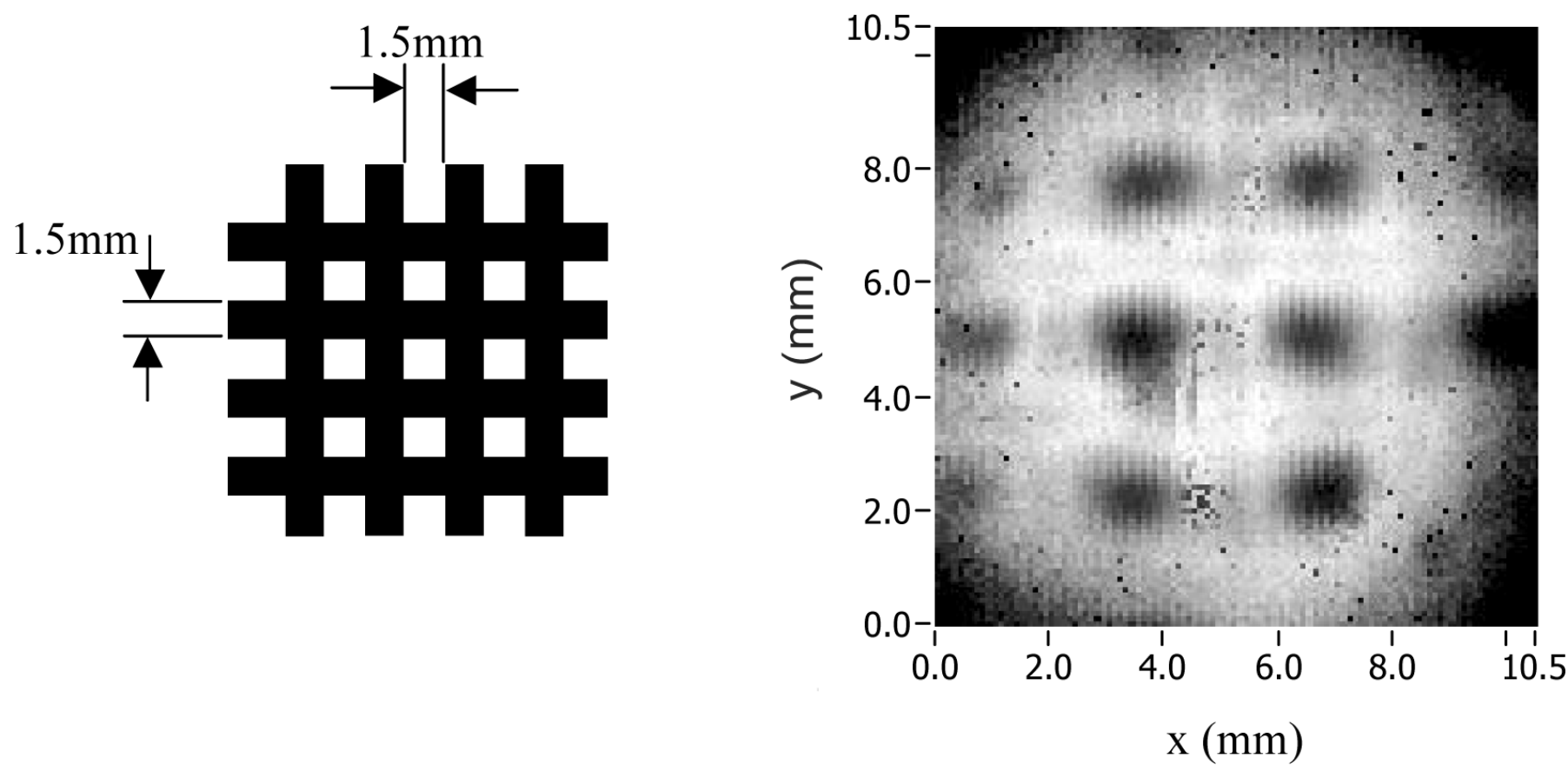

Fig. 9. Transmission ultrasound image (right) of plastic mesh target (left) using 40- $\mu \mathrm{m}$ Parylene sensor. Scan area $=10.5 \mathrm{~mm} \times 10.5 \mathrm{~mm}$, scan increments $d x=d y=0.1 \mathrm{~mm}$, photodiode aperture $=0.8 \mathrm{~mm}$.

an array with centimeter-sized apertures, 0.2 -mm element sizes and interelement spacings, and wideband $\mathrm{kPa}$ detection sensitivity. Although the concept has been demonstrated by mechanically scanning a single detector over the reflected FPI output beam, it would, in principle, be a relatively straightforward step to replace this with an optical detector array to perform the detection in parallel. This could be implemented using a photodiode or CCD array. If the latter is used, an image-intensified CCD (which can provide nanosecond exposure times) could be used to take discrete snapshots of the acoustic field distributions at successive time intervals. An equivalent and much less expensive alternative would be to use a standard relatively long exposure CCD camera in conjunction with a pulsed interrogating laser source, the latter providing the necessary temporal resolution in this configuration.

A key advantage of this type of optical sensor is the flexibility it offers in terms of the spatial sampling of the acoustic aperture. By altering the illuminating beam geometry, arbitrary array aperture shapes and sizes can be achieved, and the use of a high-element density detector array such as a CCD offers the prospect of realizing an array composed of many thousands of elements of size and spacings of the order of $10 \mu \mathrm{m}$ - a level of spatial sampling that would be prohibitively expensive, if not technically infeasible, using an array composed of discrete piezoelectric transducers. There is also a variety of configurations that can be employed to optically address the sensor. For example, a large-area FPI reflected output beam can be rapidly optically scanned over a smaller-area optical detector array using galvanometer mirrors to synthesize a larger array aperture. Alternatively, the incident beam could be focused down to a diffraction-limited spot and optically scanned over the sensor while receiving the reflected output beam with a single detector [18]. This perhaps offers the greatest flexibility in specifying the geometrical parameters of the notional array, makes the best use of the available laser power for high sensitivity, and can be readily scaled up by scanning multiple focused spots to reduce acquisition time.

The method of controlling the phase bias is key to the successful implementation of this type of sensor. Tuning the laser wavelength is conceptually ideal. However, continuously and sufficiently wide rapidly tunable lasers with sufficiently high output power $(>100 \mathrm{~mW})$ for illuminating a large area of the FPI are expensive. By contrast, the laser requirements for angle tuning are less demanding. By removing the need for wavelength tunability, the primary requirement then becomes high output power and this can readily be met using relatively inexpensive single longitudinal mode laser diodes. The situation is rather different for configurations in which a focused spot is optically scanned. Incorporating angle tuning would significantly increase the complexity of the optical system and, perhaps most importantly, angle-induced beam walk-off becomes significant when using a focused spot, particularly for higher finesse FPIs. The effect is to reduce the fringe visibility and therefore sensitivity. Wavelength tuning is therefore more appropriate when using a scanned focused spot and, due to the much reduced laser output power requirements, can be achieved at reasonable cost.

The specific nature of the optical transduction mechanism offers substantial scope to optimize the sensor performance for specific applications. For characterizing the output of ultrasound transducers or transducer arrays such as those used in diagnostic medical ultrasound, the emphasis 
may be on bandwidth and linearity, rather than sensitivity. Both of these can be improved over the values reported in this paper by reducing the polymer film thickness; e.g., a polymer-backed $10-\mu \mathrm{m}$-thick FPI would provide a 3 -dB bandwidth of $90 \mathrm{MHz}$ and, depending on the finesse, an upper limit of linear detection of several tens of MPa. If sensitivity is the issue, as is the case in biomedical photoacoustic imaging, there is substantial scope to improve this by using low-absorption, high-reflectivity dielectric coatings for the FPI mirrors to increase finesse. Recent work [8] has shown that by optimizing the ITF in this way and increasing the interrogating laser power, it is possible to achieve $0.1-\mathrm{kPa}$ detection sensitivities with a $50-\mu \mathrm{m}$ optically defined element size, a wideband sensitivity that is comparable to a 1-mm-diameter PVDF element. It is this ability to obtain high detection sensitivity with acoustically small element sizes that provides the most compelling advantage, in terms of acoustic performance, over piezoelectric methods.

Other advantages are that the sensor can be self calibrating by monitoring the ITF derivative at the FPI working point, and is electrically passive and immune to electromagnetic interference. The use of vacuum deposition methods to deposit the polymer film FPI also means that the sensor can be batch-fabricated with high repeatability at low unit cost, the latter offering the prospect of a disposable sensor head suitable for high field characterization applications where the risk of damage precludes the use of expensive piezoelectric transducer arrays.

In summary, then, this concept has the potential for use in imaging applications that require a passive receive array such as photoacoustic, electroacoustic imaging and transmission ultrasound imaging and in the characterization of fields produced by ultrasound transducers and transducer arrays such as those used in diagnostic and therapeutic medical ultrasound and industrial nondestructive testing.

\section{REFERENCES}

[1] G. Paltauf and H. Schmidt-Kloiber, "Optical method for two dimensional ultrasonic detection," Appl. Phys. Lett., vol. 75, no. 8, pp. 1048-1050, 1999.

[2] J. S. Kallman, A. E. Ashby, D. R. Ciarlo, and G. H. Thomas, "OPUS: An optically parallel ultrasound sensor," in Proc. SPIE, vol. 3912, 2000, pp. 64-73.
[3] J. D. Hamilton and M. O'Donnell, "High frequency ultrasound imaging with optical arrays," IEEE Trans. Ultrason., Ferroelect., Freq. Contr., vol. 45, no. 1, pp. 216-235, 1998.

[4] V. Wilkens and C. Koch, "Optical multilayer detection array for fast ultrasonic field mapping," Opt. Lett., vol. 24, no. 15, pp. 1026-1028, 1999.

[5] J. D. Hamilton, T. Buma, M. Spisar, and M. O'Donnell, "High frequency optoacoustic arrays using etalon detection," IEEE Trans. Ultrason., Ferroelect., Freq. Contr., vol. 47, no. 1, pp. 160-169, 2000.

[6] Y. Uno and K. Nakamura, "Pressure sensitivity of a fibre-optic microprobe for high frequency ultrasonic field," Jpn. J. Appl. Phys., vol. 38, pp. 3120-3123, 1999.

[7] P. C. Beard, F. Perennes, and T. N. Mills, "Transduction mechanisms of the Fabry Perot polymer film sensing concept for wideband ultrasound detection," IEEE Trans. Ultrason., Ferroelect., Freq. Contr., vol. 46, no. 6, pp. 1575-1582, 1999.

[8] E. Z. Zhang, B. T. Cox, and P. C. Beard, "Ultra high sensitivity, wideband Fabry Perot ultrasound sensors as an alternative to piezoelectric PVDF transducers for biomedical photoacoustic detection," in Proc. SPIE, vol. 5320, 2004, pp. 222-229.

[9] P. C. Beard, A. Hurrell, and T. N. Mills, "Characterisation of a polymer film optical fibre hydrophone for the measurement of ultrasound fields for use in the range 1-20 MHz: A comparison with PVDF needle and membrane hydrophones," IEEE Trans. Ultrason., Ferroelect., Freq. Contr., vol. 47, no. 1, pp. 256-264, 2000.

[10] P. C. Beard, F. Perennes, E. Draguioti, and T. N. Mills, "An optical fibre photoacoustic-photothermal probe," Opt. Lett., vol. 23, no. 15, pp. 1235-1237, 1998.

[11] P. C. Beard and T. N. Mills, "A 2D optical ultrasound array using a polymer film sensing interferometer," in Proc. IEEE Ultrason. Symp., 2000, pp. 1183-1186.

[12] P. C. Beard, "Photoacoustic imaging of blood vessel equivalent phantoms," in Proc. SPIE, vol. 4618, 2002, pp. 54-62.

[13] K. P. Köstli and P. C. Beard, "Two-dimensional photoacoustic imaging by use of Fourier-transform image reconstruction and a detector with an anisotropic response," Appl. Opt., vol. 42, no. 10, pp. 1899-1908, 2003.

[14] H. Yasuda, Plasma Polymerization. Orlando, FL: Academic Press, 1985.

[15] P. C. Beard, "Interrogation of Fabry Perot sensing interferometers by angle tuning," Meas. Sci. Technol., vol. 14, pp. 19982005, 2003.

[16] J. Holden, "Multiple beam interferometry: Intensity distribution in the reflected system," in Proc. Phys. Soc. Sect. B, vol. 62, pt. 7, no. 355B, 1949, pp. 405-417.

[17] J. M. Kilpatrick, W. N. MacPherson, J. S. Barton, and J. D. C. Jones, "Phase demodulation error of a fiber optic sensor with complex reflection coefficients," Appl. Opt., vol. 39, no. 9, pp. 1383-1388, 2000.

[18] B. T. Cox, E. Z. Zhang, J. G. Laufer, and P. C. Beard, "Fabry Perot polymer film fibre-optic hydrophones and arrays for ultrasound field characterisation," in J. Physics: Conf. Ser., Adv. Metrol. Ultrasound Med. (AMUM), 2004, pp. 32-37. 